\title{
Avaliação da prevalência de cárie dentária em escolares de localidade urbana da região Sudeste do Brasil
}

\section{Evaluation of the prevalence of dental caries en school-children in an urban area of southeastern Brazil}

\author{
Ben-Hur W. Moreira, Antonio C. Pereira e Sonia P. Oliveira \\ Departamento de Odontologia Social da Faculdade de Odontologia de Piracicaba-UNICAMP. \\ Piracicaba, SP - Brasil (B.H.W.M., A.C.P.), Prefeitura Municipal de Paulínia. Paulínia, SP - Brasil \\ (S.P.O.)
}

\begin{abstract}
Resumo
Realizou-se no ano de 1994, na cidade de Paulínia, Estado de São Paulo (Brasil), um levantamento epidemiológico de cárie dentária com o intuito de comparar a atual prevalência com os dados de um estudo prévio de 1980. Foram examinados 1.416 escolares de 7 a14 anos de idade, de ambos os sexos, por 10 dentistas previamente calibrados, utilizando-se os índices CPO.D e CPO.S. Verificou-se que houve uma queda da prevalência de cárie em 67,8\% em relação aos dados de 1980. Observou-se uma inversão dos componentes do índice CPO.D: em 1980 prevalecia o componente cariado (69,5\%), enquanto que o componente obturado prevaleceu em 1994 (79,0\%). Os componentes extraídos e a extração indicada praticamente desapareceram no ano de 1994.
\end{abstract}

Cárie dentária, epidemiologia. Integração docente-assistencial. Avaliação de programas.

\begin{abstract}
s
An epidemiologic survey of dental caries was undertaken in the town of Paulinia, S. Paulo state, south-eastern Brazil, in 1994, for the purpose of comparing the present prevalence with that registered during the previous study of 1980. The children examined numbered 1,416, were of both sexes and aged 7-14. Ten dentists, preciously trained, used DMF.T and DMF.S dental caries indexes. A level of prevalence of dental caries $67.8 \%$ below that of 1980 was found. Futher, there had been an inversion of DMF.T index components. Whereas in 1980 the component caries was higher (69.5\%), while the filing component prevailed (79.0\%) in 1994. The missing toth component has disappeared altogether in this recent survey.
\end{abstract}

Dental caries, epidemiology. Teaching care integration. Program evaluation.

Correspondência para / Correspondence to: Antonio C. Pereira - Departamento de Odontologia Social da Faculdade de Odontologia de Piracicaba. Av. Limeira, 901 - 13414-900 Piracicaba, SP - Brasil. Fax: (0194) 21-0063.

Edição subvencionada pela FAPESP. Processo 95/2290-6.

Recebido em 24.2.1995. Reapresentado em 29.4.1996. Aprovado em 3.5.1996. 


\section{INTRODUÇÃO}

Os estágios extramurais nas Faculdades de Odontologia devem ser obrigatórios, pois fazem parte do "curriculum" mínimo dos cursos de graduação e estão amparados pela Lei Federal no 6.494 de 7/12/ 1977, pelo Decreto Federal no 87.497 de 18/8/1982 e pelas resoluções $n^{\circ} 04$ de 3/9/1982 e 116 de 25/8/ 1984 do Conselho Federal de Odontologia.

A participação de estudantes do curso de graduação ( $7^{\circ}$ e $8^{\circ}$ semestres) da Faculdade de Odontologia de Piracicaba, Universidade Estadual de Campinas, em programas de integração docenteassistencial (estágios extramurais) iniciou-se em 1970, logo após a assinatura de um convênio com a Prefeitura Municipal de Piracicaba, SP, em 1969, para atendimento odontológico da zona rural desse mesmo município.

Em 1975 foi iniciado um segundo programa de integração docente-assistencial, programa esse incipiente, na cidade de Paulínia, Estado de São Paulo, distante $60 \mathrm{~km}$ de Piracicaba. Esse programa foi reformulado em 1980 e, posteriormente, em 1983, quando surgiu o Programa Integrado de Educação e Saúde Escolar (PIESE) ${ }^{6}$ e que sofreu, em 1985, novas alterações, com modificações também no equipamento simplificado utilizado. O programa foi realizado no Centro Odontológico Municipal (COM), que conta com a participação de dois cirurgiões dentistas na coordenação dos programas, dois cirurgiões dentistas na supervisão dos estagiários, três cirurgiões dentistas no atendimento a pré-escolares, gestantes e adultos, uma THD* e 14 ACDs**.

Alguns artigos sobre o assunto já foram publicados e neles são analisados os tipos de estágios, os objetivos e os dados de produtividade $3,4,5,6,10$.

Em 1980 foi realizado um levantamento epidemiológico de cárie dentária na população escolar de Paulínia. Um segundo levantamento foi realizado em 1994.

O presente trabalho tem por objetivo comparar e analisar os dados desses levantamentos.

\section{MATERIAL E MÉTODO}

Paulínia é uma cidade do interior do Estado de São Paulo, dista $110 \mathrm{~km}$ de São Paulo, a capital do Estado, possuindo atualmente cerca de 45.000 habitantes, nela fica instalada uma das maiores refinarias de petróleo do País, a Refinaria do Planalto (REPLAN). A água de abasteci- mento público é fluoretada com o ácido fluorsilícico (0,7ppmF), desde 1980 .

Para os levantamentos realizados foram utilizados o Índice CPO.S proposto por Klein e col. ${ }^{2} \mathrm{e}$, posteriormente, foi calculado CPO.D proposto por Klein e Palmer ${ }^{1}$.

Os inquéritos foram realizados por examinadores previamente calibrados, sendo 20 em 1980 e 10 em 1994.

Foram utilizadas sondas exploradoras e espelhos bucais número 5 (sem aumento), sendo que o exame foi realizado com luz natural nos pátios das escolas. A mesma metodologia foi utilizada nos estudos de 1980 e 1994.

Os dados dos exames foram anotados em fichas individuais, utilizando-se códigos e critérios preconizados pela Disciplina de Odontologia Preventiva e Saúde Pública da Faculdade de Odontologia de Piracicaba.

Em 1980 foram examinados 1.156 escolares da rede estadual de ensino, idade entre 7 e 14 anos de idade, ambos os sexos (566 do sexo masculino e 590 do sexo feminino). Em 1994 foram examinados 1.416 escolares de ambos os sexos (666 do sexo masculino e 750 do sexo feminino). Esses números de escolares examinados representavam cerca de $16 \%$ da população dessa faixa etária, tanto em 1980 como em 1994.

\section{RESULTADOS E DISCUSSÃO}

Verificamos, pela Tabela 1, que os escolares, em 1980, apresentavam alta prevalência da cárie dentária, sendo o componente cariado $(\mathrm{C})$ o principal responsável por esses índices elevados: aos 7 anos de idade o Índice CPO.D já era 2,4, sendo 1,9 cariado; aos 10 anos, de 5,1 CPO.D, 3,6 dentes eram cariados; aos 12 anos, dos 8,2 CPO.D, 5,5 eram dentes cariados. O componente cariado correspondia a $79,2 \%$ do Índice CPO.D aos 7 anos de idade, 70,6\% aos 10 anos e a 58,0\% aos 12 anos (Tabela 2).

Em relação ao total de escolares, $72,9 \%$ do Índice CPO.D era constituído pelos componentes $\mathrm{Ce}$ Ei, isto é, por tratamento a realizar, e apenas $27,1 \%$ era constituído pelos componentes $\mathrm{E}$ e O, isto é, por tratamento realizado, revelando que essa população era pouco assistida em relação à odontologia. Esses resultados são semelhantes aos encontrados por Rosa e col. ${ }^{9}$, na cidade de São José dos Campos, SP, em 1979.

Em 1994, o Índice CPO.D variou de 0,4 aos 7 anos de idade até o máximo de 4,3 aos 14 anos. O principal componente responsável passou a ser o restaurado (0), que variou de 0,3 aos 7 anos até 3,4 aos 14 anos de idade; esse componente foi o responsável por 79,0\% do Índice CPO.D em todas as idades; já o dente cariado (C) foi o responsável por $21,0 \%$ do Índice CPO.D, em todas as idades.

* THD - Técnico em higiene dentária

**ACD - Assistente de consultório dentário 
Tabela 1 - Índice CPO.D médio e componentes de escolares de ambos os sexos, segundo a idade, Paulínia, SP, 1980 e 1994.

\begin{tabular}{|c|c|c|c|c|c|c|c|}
\hline Idade & Ano & $\mathrm{N}$ & $\mathrm{C}$ & $\mathrm{Ei}$ & $E$ & $\mathrm{O}$ & CPO.D \\
\hline \multirow[t]{2}{*}{7} & 1980 & 104 & 1,9 & - & - & 0,5 & 2,4 \\
\hline & 1994 & 221 & 0,1 & - & - & 0,3 & 0,4 \\
\hline \multirow[t]{2}{*}{8} & 1980 & 178 & 2,5 & 0,1 & - & 0,5 & 3,1 \\
\hline & 1994 & 194 & 0,4 & - & - & 0,4 & 0,8 \\
\hline \multirow[t]{2}{*}{9} & 1980 & 200 & 2,8 & 0,2 & 0,3 & 0,7 & 4,0 \\
\hline & 1994 & 182 & 0,3 & - & - & 0,8 & 1,1 \\
\hline \multirow[t]{2}{*}{10} & 1980 & 175 & 3,6 & 0,2 & 0,4 & 0,9 & 5,1 \\
\hline & 1994 & 212 & 0,4 & - & - & 1,0 & 1,4 \\
\hline \multirow[t]{2}{*}{11} & 1980 & 175 & 5,0 & 0,3 & 0,5 & 0,8 & 6,6 \\
\hline & 1994 & 163 & 0,5 & - & - & 2,0 & 2,5 \\
\hline \multirow[t]{2}{*}{12} & 1980 & 173 & 5,5 & 0,4 & 0,5 & 1,8 & 8,2 \\
\hline & 1994 & 184 & 0,6 & - & - & 2,4 & 3,0 \\
\hline \multirow[t]{2}{*}{13} & 1980 & 113 & 6,5 & 0,3 & 0,8 & 3,1 & 10,7 \\
\hline & 1994 & 163 & 0,7 & - & - & 3,0 & 3,7 \\
\hline \multirow[t]{2}{*}{14} & 1980 & 38 & 8,3 & 0,4 & 1,2 & 4,4 & 14,3 \\
\hline & 1994 & 97 & 0,8 & - & 0,1 & 3,4 & 4,3 \\
\hline \multirow[t]{2}{*}{ Total } & 1980 & 1.156 & 4,1 & 0,2 & 0,4 & 1,2 & 5,9 \\
\hline & 1994 & 1.416 & 0,4 & - & - & 1,5 & 1,9 \\
\hline
\end{tabular}

O componente perdido (dentes com extração indicada + dentes extraídos) desapareceu quase que totalmente; apenas na idade de 14 anos foi encontrado $2,3 \%$ do componente extraído.

Observando-se o Índice CPO.D aos 12 anos de idade, em 1994, verificou-se que esses escolares já atingiram a meta prevista pela $\mathrm{OMS}^{7}$, para o ano 2000, (Índice CPO.D no máximo igual a 3,0), e têm um índice de cárie semelhante ao de escolares dessa idade em alguns países, tais como, Dinamarca, Itália, Noruega e Reino Unido ${ }^{8}$, cujos índices CPO.D foram, respectivamente, 3,0, 3,0, 2,9 e 3,1 .

Dentro da escala de severidade da cárie dentária proposta pela $\mathrm{OMS}^{7}$, a prevalência desse problema, para os escolares de 12 anos de idade de Paulínia, passou de muito alta em 1980 para uma prevalência moderada em 1994.

Os primeiros molares permanentes são os principais dentes das arcadas dentárias por serem considerados a "chave da oclusão". Devido à sua morfologia, principalmente na superfície oclusal, são os dentes mais susceptíveis à cárie dentária e, por conseguinte, são os primeiros dentes a serem extraídos. É, portanto, necessário empregar os mais variados métodos de prevenção, evitando-se a perda e, geralmente, perda precoce desses dentes.

Pela Tabela 3 pode-se observar que, em 1980, os escolares apresentavam, em média, 3,4 primeiros molares permanentes atacados pela cárie, sendo o componente cariado responsável por $64,7 \%$ da composição do Índice CPO.D para esses dentes; o com-

Tabela 2 - Composição percentual do Índice CPO.D de escolares de ambos os sexos, segundo a idade, Paulínia, SP, 1980 e 1994

\begin{tabular}{|c|c|c|c|c|c|c|}
\hline Idade & Ano & $\mathrm{C}$ & $\mathrm{Ei}$ & $\mathrm{E}$ & $\mathrm{O}$ & CPO.D \\
\hline \multirow[t]{2}{*}{7} & 1980 & 79,2 & - & - & 20,8 & 100,0 \\
\hline & 1994 & 25,0 & - & - & 75,0 & 100,0 \\
\hline \multirow[t]{2}{*}{8} & 1980 & 80,7 & 3,2 & - & 16,1 & 100,0 \\
\hline & 1994 & 50,0 & - & - & 50,0 & 100,0 \\
\hline \multirow[t]{2}{*}{9} & 1980 & 70,0 & 5,0 & 7,5 & 17,5 & 100,0 \\
\hline & 1994 & 27,3 & - & - & 72,7 & 100,0 \\
\hline \multirow[t]{2}{*}{10} & 1980 & 70,6 & 3,9 & 7,8 & 17,7 & 100,0 \\
\hline & 1994 & 28,6 & - & - & 71,4 & 100,0 \\
\hline \multirow[t]{2}{*}{11} & 1980 & 75,8 & 4,5 & 7,6 & 12,1 & 100,0 \\
\hline & 1994 & 20,0 & - & - & 80,0 & 100,0 \\
\hline \multirow[t]{2}{*}{12} & 1980 & 67,1 & 4,9 & 6,1 & 21,9 & 100,0 \\
\hline & 1994 & 20,0 & - & - & 80,0 & 100,0 \\
\hline \multirow[t]{2}{*}{13} & 1980 & 60,7 & 2,8 & 7,5 & 29,0 & 100,0 \\
\hline & 1994 & 18,9 & - & - & 81,1 & 100,0 \\
\hline \multirow[t]{2}{*}{14} & 1980 & 58,0 & 2,8 & 8,4 & 30,8 & 100,0 \\
\hline & 1994 & 18,6 & - & 2,3 & 79,1 & 100,0 \\
\hline \multirow[t]{2}{*}{ Total } & 1980 & 69,5 & 3,4 & 6,8 & 20,3 & 100,0 \\
\hline & 1994 & 21,0 & - & - & 79,0 & 100,0 \\
\hline
\end{tabular}


ponente restaurado por apenas $17,6 \%$ e o perdido (Ei + E) era responsável por $17,7 \%$.

Em 1994 houve total inversão na composição do Índice CPO.D para primeiros molares permanentes: $81,2 \%$ para o componente restaurado, $18,8 \%$ para o cariado e o componente perdido desapareceu.

Na Figura 1 pode-se observar os Índices CPO.D dos escolares, segunda a idade tanto em 1980 como em 1994.

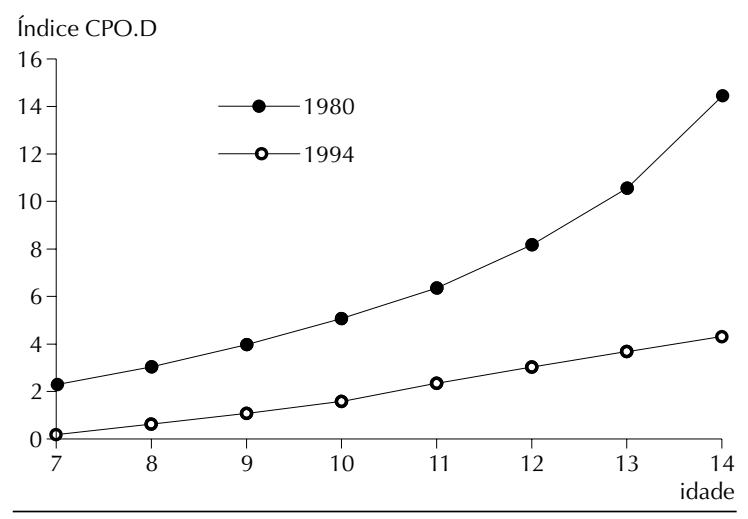

Figura 1 - Índice CPO.D médio de escolares, ambos os sexos, segundo a idade. Paulínia, SP, 1980 e 1994.

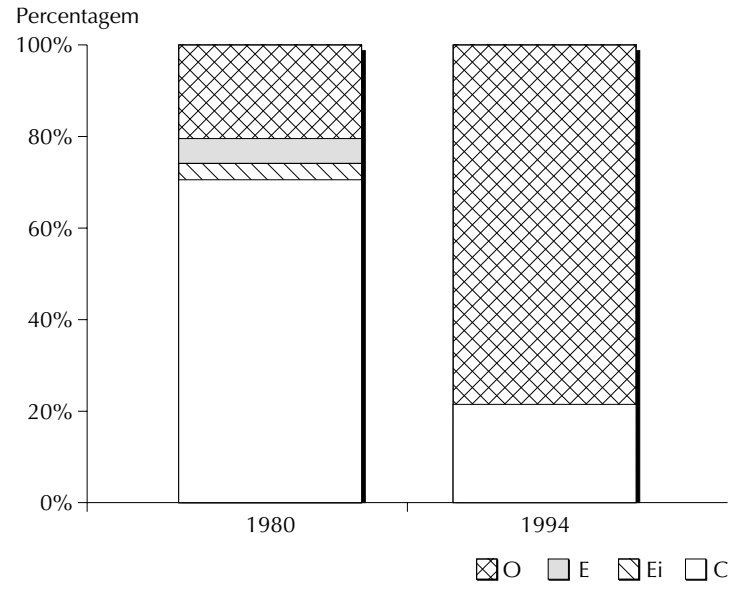

Figura 2 - Composição percentual do Índice CPO.D, ambos os sexos, todas as idades. Paulínia, SP,1980 e 1994.

Em relação aos totais da Tabela 2 pode-se observar esses dados esquematizados na Figura 2.

Comparando-se os dados de 1980 com os de 1994, para todas as idades, verificou-se que houve:

a) redução do índice CPO.D de 5,9 para 1,9, isto é, $67,8 \%$ de redução da cárie dentária;

b) redução do incremento médio de cárie em todas as idades de 1,7 para 0,6 , isto é, $64,7 \%$ de redução; c) redução drástica da composição percentual das necessidades a serem tratadas $(\mathrm{C}+\mathrm{Ei}) \mathrm{de}$ $72,9 \%$ para $21,0 \%$;

d) aumento significativo da composição percentual das necessidades tratadas $(\mathrm{E}+\mathrm{O}) \mathrm{de}$ $27,1 \%$ para $79,0 \%$;

e) redução significante da composição percentual do componente dos dentes extraídos e com extração indicada de $10,2 \%$ para zero.

Comparando-se os dados relativos aos primeiros molares permanentes de 1980 com os de 1994, verificamos que houve:

a) redução do ataque de cárie de 3,4 para 1,6, isto é, $52,9 \%$ de redução;

b) redução drástica da composição percentual das necessidades a serem tratadas $(\mathrm{C}+\mathrm{Ei}) \mathrm{de}$ $70,6 \%$ para $18,8 \%$;

c) aumento significativo da composição percentual das necessidades tratadas $(\mathrm{E}+\mathrm{O}) \mathrm{de}$ $29,4 \%$ para $81,2 \%$;

d) redução da composição percentual dos dentes extraídos e com extração indicada de 17,7\% para zero.

\section{CONSIDERAÇÕES FINAIS}

A excelente redução da cárie dentária, nesse período, deve-se não somente à fluoretação da água de abastecimento, mas também às aplicações tópicas de flúor, às aplicações de selantes de fissuras nos dois últimos anos, ao ensino da técnica correta de higiene dentária, à educação para a saúde (palestras) e ao tratamento odontológico, tarefas estas realizadas pelo PIESE e demais programas vinculados ao sistema prestador de serviço odontológico ao Município de Paulínia.

Tabela 3 - Ataque de cáries nos primeiros molares permanentes em escolares de ambos os sexos, segundo o ano do levantamento, Paulínia, SP.

\begin{tabular}{lcrcr}
\hline \multirow{2}{*}{$\begin{array}{l}\text { Índice CPO.D e } \\
\text { componentes }\end{array}$} & \multicolumn{2}{c}{1980} & \multicolumn{2}{c}{1994} \\
\cline { 2 - 5 } & Média & \multicolumn{1}{c}{$\%$} & Média & \multicolumn{1}{c}{$\%$} \\
\hline C & 2,2 & 64,7 & 0,3 & 18,8 \\
Ei & 0,2 & 5,9 & 0,0 & 0,0 \\
E & 0,4 & 11,8 & 0,0 & 0,0 \\
O & 0,6 & 17,6 & 1,3 & 81,2 \\
\hline CPO.D & 3,4 & 100,0 & 1,6 & 100,0 \\
\hline
\end{tabular}

É de se esperar que a incidência da cárie dentária em escolares de Paulínia continue a diminuir, pois novos programas estão sendo implantados e executados nestes dois últimos anos, tais como, programa preventivo aos escolares realizados nas próprias es- 
colas, programa preventivo aos pré-escolares da faixa etária de 3 a 6 anos de idade, e programa preventivo para a faixa etária de 0 a 2 anos de idade, realizado nas creches.

Através destes dados podemos verificar a importância dos estágios extramurais para estudantes de

\section{REFERÊNCIAS BIBLIOGRÁFICAS}

1. KLEIN, H. \& PALMER, C. E. Dental caries in American indian children. In: Chaves, M. M. Odontologia social. $2^{\mathrm{a}}$ ed. Rio de Janeiro, Edit. Labor do Brasil S.A., 1977. p. 34.

2. KLEIN et al. apud. Chaves, M. M. Odontologia social. $2^{\mathrm{a}}$ ed. Rio de Janeiro, Edit. Labor do Brasil S.A., 1977. p. 35.

3. MOREIRA, B. H. W. et al. Participação de estudantes de odontologia em programas de integração docenteassistencial. Rev. Bras. Odont., 42: 30-6, 1985.

4. MOREIRA, B. H. W. \& OLIVEIRA, S. P. Integração docente-assistencial: análise dos resultados de um programa. Rev. Gaúcha Odont., 35: 284-6, 1987.

5. MOREIRA, B. H. W. \& OLIVEIRA, S. P. Programa de integração docente-assistencial. II. Análise dos resultados de 1985 e 1996. Rev. Bras. Odont., 45: 24-32, 1988. odontologia, principalmente quando esses estágios são bem programados, executados e avaliados. Cada estudante de odontologia é obrigado a cumprir 80 horas por semestres nesses estágios. As esquipes são constituídas de 8 estagiários por semana, que trabalham em odontologia a quatro mãos.

6. OLIVEIRA, S.P. et al. Programa integrado de educação e saúde escolar. Rev. Gaúcha Odont., 34: 254-66, 1986.

7. ORGANIZAÇÃO MUNDIAL DA SAÚDE apud. PINTO, V. G. Odontologia social e preventiva. $2^{\mathrm{a}}$ ed., São Paulo, Livr. Edit. Santos, 1990. p. 135.

8. PINTO, V. G. Saúde bucal: panorama internacional. Brasília, Ministério de Saúde, 1990.

9. ROSA, A. G. F. et al. Avaliação da assistência odontológica no serviço local de saúde de São José dos Campos, S.P. Divulgação Saúde Debate (6): 55-60, 1991.

10. TUMANG, A. J. \& MOREIRA, B. H. W. Integração docente-assistencial em um programa odontológico para escolares da zona rural de Piracicaba. I. Resultados de 1970/ 74. Rev. Ass. Paul. Cirur. Dent., 33:440-6, 1979. 